\title{
Theory and Phenomenology of Composite 2-Higgs Doublet Models
}

\author{
Stefania De Curtis \\ INFN, Sezione di Firenze, and Department of Physics and Astronomy, University of Florence, \\ Via G. Sansone 1, 50019 Sesto Fiorentino, Italy \\ E-mail: decurtisefi.infn.it
}

\section{Stefano Moretti}

School of Physics and Astronomy, University of Southampton, Southampton, SO17 1BJ, United Kingdom

E-mail: S.Morettiesoton.ac.uk

\section{Kei Yagyu}

INFN, Sezione di Firenze, and Department of Physics and Astronomy, University of Florence,

Via G. Sansone 1, 50019 Sesto Fiorentino, Italy

E-mail: yagyuefi.infn.it

\section{Emine Yildirim*}

School of Physics and Astronomy, University of Southampton, Southampton, SO17 1BJ, United Kingdom

E-mail: eylg13esoton.ac.uk

In this talk, we report unitarity constraints and phenomenological studies at the Large Hadron Collider for the extra Higgs bosons of a Composite 2-Higgs Doublet Model.

Prospects for Charged Higgs Discovery at Colliders 3-6 October 2016

Uppsala, Sweden

\footnotetext{
* Speaker.
} 


\section{Introduction}

After the discovery of a Higgs like state at the mass of $125 \mathrm{GeV}$ at the Large Hadron Collider (LHC) in July 2012 [1, 2], understanding the origin of Electro-Weak Symmetry Breaking (EWSB) is one of the primary tasks in particle physics. EWSB in Beyond the Standard Models (BSM) scenarios can occur in two ways: through a weakly interacting model (with elementary Higgs bosons) or a strongly interacting one (with composite Higgs bosons). A composite Higgs state arising as a pseudo Nambu-Goldstone Boson (pNGB), from a new dynamics at the compositeness scale $f$, can lead to EWSB, as a result of couplings of the Higgs sector to the SM. Therefore a remarkable way to generate new light (pseudo)scalars in the spectrum is to make them also pNGBs. We study here a Composite 2-Higgs Doublet Model (C2HDM) [3], wherein two Higgs fields, $\Phi_{1}$ and $\Phi_{2}$, eventually inducing a SM-like Higgs state $(h)$, a CP-even field $(H)$, a CP-odd one $(A)$ and a charged pair $\left(H^{ \pm}\right)$, are introduced.

Perturbative unitarity bounds of a C2HDM based on $S O(6) \rightarrow S O(4) \times S O(2)$ coset structure will be reported upon by using perturbative unitarity to cover all energies accessible at the LHC. The pNGB nature of the Higgses results in a modification of their couplings to matter with respect to the E2HDM (Elementary 2HDM) case and, consequently, the scattering amplitudes are left with a non-vanishing $s$-dependence. Here we will not present the Higgs potential generated by radiative corrections. Instead, we will assume for it the same structure as in the E2HDM, see our work in Ref. [4]. Moreover, in [5], we have studied LHC phenomenology of different Yukawa types of a C2HDM. Herein, the theoretical and experimental constraints on the parameter space of C2HDMs and E2HDMs showed differences and the latter will guide us in the phenomenological study of a C2HDM versus a E2HDM.

This report is organized as follows. In Section II the C2HDM based on $S O(6) / S O(4) \times S O(2)$ will be discussed. In section III, unitarity bounds will be discussed. In section IV, the LHC phenomenology of $\mathrm{C} 2 \mathrm{HDMs}$ is presented while conclusions are given in section $\mathrm{V}$.

\section{Effective Lagrangian in the C2HDM}

\subsection{Kinetic Lagrangian and Gauge Couplings}

In general, once the coset space has been chosen, the low-energy Lagrangian is fixed at the two-derivative level, the basic ingredient being the pNGB matrix which transforms non-linearly under the global group.

The kinetic Lagrangian invariant under the $S O(6)$ symmetry can be constructed by the analogue of the construction in non-linear sigma models developed in [6], as

$$
\mathscr{L}_{\text {kin }}=\frac{f^{2}}{4}\left(d_{\alpha}^{\hat{a}}\right)_{\mu}\left(d_{\alpha}^{\hat{a}}\right)^{\mu}, \text { with }\left(d_{\alpha}^{\hat{a}}\right)_{\mu}=i \operatorname{tr}\left(U^{\dagger} D_{\mu} U T_{\alpha}^{\hat{a}}\right), \text { where } \alpha=1,2 . \quad \hat{a}=1,4 .
$$

Here $U$ is the pNGB matrix:

$$
U=\exp \left(i \frac{\Pi}{f}\right), \text { with } \Pi \equiv \sqrt{2} h_{\alpha}^{\hat{a}} T_{\alpha}^{\hat{a}}=-i\left(\begin{array}{ccc}
0 \times 4 & h_{1}^{\hat{a}} & h_{2}^{\hat{a}} \\
-h_{1}^{\hat{a}} & 0 & 0 \\
-h_{2}^{\hat{a}} & 0 & 0
\end{array}\right) .
$$


In Eq. (2.1), the covariant derivative $D_{\mu}$ is given by

$$
D_{\mu}=\partial_{\mu}-i g T_{L}^{a} W_{\mu}^{a}-i g^{\prime} Y B_{\mu}, \quad \text { where } T^{\hat{a}}, T_{L}^{a} \text { and } Y \text { are } S O(6) \text { generators. }
$$

Some coefficients of the $\Phi V V$ and $\Phi \Phi V\left(V=W^{ \pm}, Z\right)$ couplings are given by

$h W^{+} W^{-}=g m_{W}\left(1-\frac{\xi}{2}\right) \cos \theta, \quad H W^{+} W^{-}=-g m_{W}\left(1-\frac{\xi}{2}\right) \sin \theta$,

$H^{+} \partial^{\mu} H W_{\mu}^{-}=\mp i \frac{g}{2}\left(1-\frac{5}{6} \xi\right) \cos \theta, \quad H^{+} \partial^{\mu} h W_{\mu}^{-}=\mp i \frac{g}{2}\left(1-\frac{5}{6} \xi\right) \sin \theta$,

$A \partial^{\mu} h Z_{\mu}=-\frac{g_{z}}{2}\left(1-\frac{5}{6} \xi\right) \sin \theta$, where $\xi=v_{\mathrm{SM}}^{2} / f^{2}$ ( $\theta$ is the mixing angle between CP-even states).

\subsection{Yukawa Lagrangian}

The low-energy (below the scale $f$ ) Yukawa Lagrangian is constructed by determining the embedding scheme of the SM fermions into $S O(6)$ multiplets. This embedding can be explained via the mechanism based on the partial compositeness hypothesis [7] by mixing elementary SM fermions with composite fermions in the invariant form under the SM SU $(2)_{L} \times U(1)_{Y}$ gauge symmetry. The Yukawa Lagrangian is then given in terms of the 15-plet of pNGB fields $\Sigma$ and the 6-plet of fermions defined as [5]

$$
\mathscr{L}_{Y}=f\left[\bar{Q}_{L}^{u}\left(a_{u} \Sigma-b_{u} \Sigma^{2}\right) U_{R}+\bar{Q}_{L}^{d}\left(a_{d} \Sigma-b_{d} \Sigma^{2}\right) D_{R}+\bar{L}_{L}\left(a_{e} \Sigma-b_{e} \Sigma^{2}\right) E_{R}\right]+\text { h.c. }
$$

We derive our Yukawa Lagrangian up to the squared power of $\Sigma$, since the terms with the cubic and more than cubic power of $\Sigma$ do not generate any extra distinct contribution to it. After the Yukawa Lagrangian is described in terms of the complex doublet Higgs fields $\Phi_{1}$ and $\Phi_{2}$, Flavor Changing Neutral Currents (FCNCs) occur in C2HDMs, as seen already in E2HDMs. In order to prevent FCNCs, we implement a $C_{2}$ symmetry transformation [3] as follows:

$$
U\left(\pi_{1}^{\hat{a}}, \pi_{2}^{\hat{a}}\right) \rightarrow C_{2} U\left(\pi_{1}^{\hat{a}}, \pi_{2}^{\hat{a}}\right) C_{2}=U\left(\pi_{1}^{\hat{a}},-\pi_{2}^{\hat{a}}\right),
$$

where

$$
C_{2}=\operatorname{diag}(1,1,1,1,1,-1) .
$$

and then interaction Lagrangian becomes:

$$
\begin{aligned}
\mathscr{L}_{Y} & =\sum_{f=u, d, e} \frac{m_{f}}{v_{\mathrm{SM}}} \bar{f}\left(\bar{X}_{f}^{h} h+\bar{X}_{f}^{H} H-2 i I_{f} \bar{X}_{f}^{A} \gamma_{5} A\right) f \\
& +\frac{\sqrt{2}}{v_{\mathrm{SM}}} \bar{u} V_{u d}\left(m_{d} \bar{X}_{d}^{A} P_{R}-m_{u} \bar{X}_{u}^{A} P_{L}\right) d H^{+}+\frac{\sqrt{2}}{v_{\mathrm{SM}}} \bar{v} m_{e} \bar{X}_{e} P_{R} e H^{+}+\text {h.c. }
\end{aligned}
$$

The definition of $\zeta_{h, H, A}$ and $\xi_{h, H, A}$ are given by (at the first order in $\xi$ ):

$$
\begin{aligned}
& \zeta_{h}=\left(1-\frac{3}{2} \xi\right) c_{\theta}+s_{\theta} \cot \beta, \quad \xi_{h}=\left(1-\frac{\xi}{2}\right) c_{\theta}-s_{\theta} \tan \beta \\
& \zeta_{H}=-\left(1-\frac{3}{2} \xi\right) s_{\theta}+c_{\theta} \cot \beta, \quad \xi_{H}=-\left(1-\frac{\xi}{2}\right) s_{\theta}-c_{\theta} \tan \beta \\
& \zeta_{A}=\left(1+\frac{\xi}{2}\right) \cot \beta, \quad \xi_{A}=-\left(1-\frac{\xi}{2}\right) \tan \beta
\end{aligned}
$$




\begin{tabular}{c||ccc||ccc||ccc|ccc|ccc}
\hline \hline & $U_{R}$ & $D_{R}$ & $E_{R}$ & $\left(a_{u}, b_{u}\right)$ & $\left(a_{d}, b_{d}\right)$ & $\left(a_{e}, b_{e}\right)$ & $\bar{X}_{u}^{h}$ & $\bar{X}_{d}^{h}$ & $\bar{X}_{e}^{h}$ & $\bar{X}_{u}^{H}$ & $\bar{X}_{d}^{H}$ & $\bar{X}_{e}^{H}$ & $\bar{X}_{u}^{A}$ & $\bar{X}_{d}^{A}$ & $\bar{X}_{e}^{A}$ \\
\hline Type-I & - & - & - & $(0, \sqrt{ })$ & $(0, \sqrt{ })$ & $(0, \sqrt{ })$ & $\zeta_{h}$ & $\zeta_{h}$ & $\zeta_{h}$ & $\zeta_{H}$ & $\zeta_{H}$ & $\zeta_{H}$ & $\zeta_{A}$ & $\zeta_{A}$ & $\zeta_{A}$ \\
\hline Type-II & - & + & + & $(0, \sqrt{ })$ & $(\sqrt{ }, 0)$ & $(\sqrt{ }, 0)$ & $\zeta_{h}$ & $\xi_{h}$ & $\xi_{h}$ & $\zeta_{H}$ & $\xi_{H}$ & $\xi_{H}$ & $\zeta_{A}$ & $\xi_{A}$ & $\xi_{A}$ \\
\hline Type-X & - & - & + & $(0, \sqrt{ })$ & $(0, \sqrt{ })$ & $(\sqrt{ }, 0)$ & $\zeta_{h}$ & $\zeta_{h}$ & $\xi_{h}$ & $\zeta_{H}$ & $\zeta_{H}$ & $\xi_{H}$ & $\zeta_{A}$ & $\zeta_{A}$ & $\xi_{A}$ \\
\hline Type-Y & - & + & - & $(0, \sqrt{ })$ & $(\sqrt{ }, 0)$ & $(0, \sqrt{ })$ & $\zeta_{h}$ & $\xi_{h}$ & $\zeta_{h}$ & $\zeta_{H}$ & $\xi_{H}$ & $\zeta_{H}$ & $\zeta_{A}$ & $\xi_{A}$ & $\zeta_{A}$ \\
\hline \hline
\end{tabular}

Table 1: Charge assignment for right-handed fermions under the $C_{2}$ symmetry in C2HDMs. All the lefthanded fermions $Q_{L}^{u}, Q_{L}^{d}$ and $L_{L}$ are transformed as even under $C_{2}$.

In the limit of $\xi \rightarrow 0$, these coefficients get the same form as the corresponding ones in a softlybroken (by a mass term $M$ ) $Z_{2}$ symmetric version of the E2HDM [8]. See Tab. 1 for the available Yukawa types of a $2 \mathrm{HDM}$.

\section{Unitarity Bounds}

In order to calculate the bounds from perturbative unitarity in our C2HDMs, all possible 2-to2-body bosonic elastic scatterings are considered. Our conclusive results have been presented in [4]. We use the same method as the elementary models such as the SM [9] and E2HDM [10, 11, $12,13,14]$ to find such unitarity bounds. For example, we implement the following condition [15] for each eigenvalue of the $S$-wave amplitude matrix:

$$
\left|\operatorname{Re}\left(x_{i}\right)\right| \leq 1 / 2 .
$$

The most important difference between the unitarity bounds in a E2HDM and a C2HDM is the presence of a squared energy dependence in the $S$-wave amplitude of the latter, since in C2HDMs the Higgs-Gauge-Gauge couplings is modified from that in E2HDMs by the factor $(1-\xi)$. The energy dependence of the $S$-wave amplitudes cause unitarity violation and asks for an Ultra-Violet (UV) completion of the C2HDM. However, we stay in the energy region where C2HDMs and E2HDMs are both unitarity in order to compare the bounds on the mass of the extra Higgs bosons. In Fig.1, one of the main result is that there is an upper limit on $\sqrt{s}$ for the no-mixing $\cos \theta=1$ case, while there is an upper limit on both $\sqrt{s}$ and $m_{\Phi}$ for non-zero mixing $\cos \theta=0.99$ case.

\section{LHC phenomenology}

We first study the constraints on the parameter space of C2HDMs from searches for extra Higgs bosons at collider experiments and significant differences among the various Yukawa types are reported for the different values of $\xi=0.1,0.07,0.04$ and 0 (which is E2HDM case) [5]. We then investigate, over the accessible regions of parameter space, the deviations of the SM-like Higgs $(h)$ couplings from the SM values. The scaling factors $\kappa_{X}$ for the $h X X$ couplings are given by $\kappa_{X}=g_{h X X}^{\mathrm{NP}} / g_{h X X}^{\mathrm{SM}}$ and, in the C2HDM, these are given at the tree level by

$$
\kappa_{V}=\left(1-\frac{\xi}{2}\right) c_{\theta}(V=W, Z), \quad \kappa_{f}=\bar{X}_{f}^{h}=\zeta_{h} \text { or } \xi_{h}(f=u, d, e) .
$$



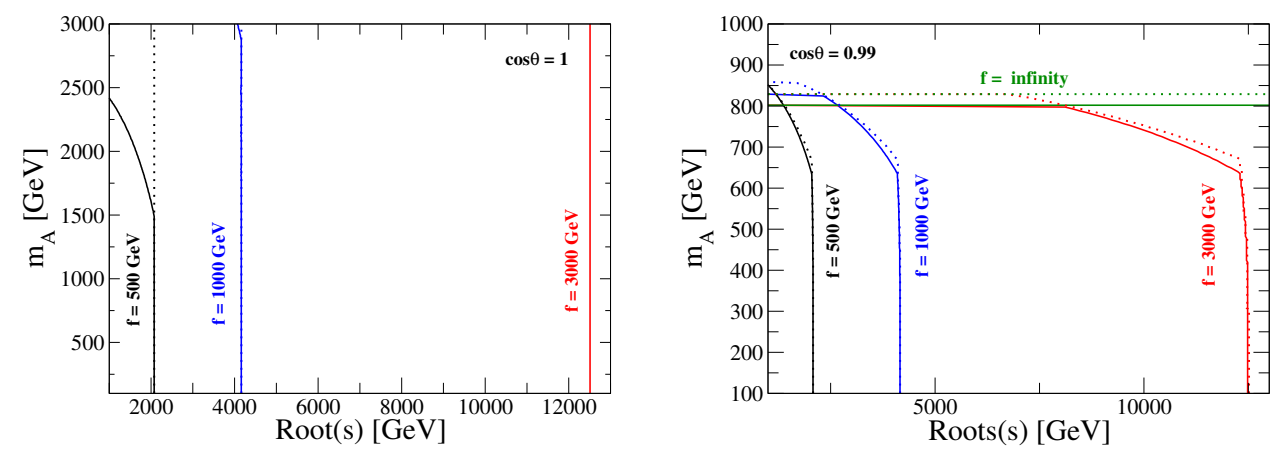

Figure 1: Constraints from unitarity and vacuum stability in the case of $\tan \beta=1$ and $m_{H^{ \pm}}=m_{A}$ for several fixed values of $f . m_{H}=m_{A}$ for the solid curves, $m_{H}=m_{A} \pm 500 \mathrm{GeV}$ for the dashed curves. For all the plots, $M$ is scanned. $f \rightarrow \infty$ is E2HDM case.

In C2HDMs non-zero values of both $\xi$ and $\theta$ can give $\kappa_{X} \neq 1$, while in E2HDMs only that of $\theta$ can give $\kappa_{X} \neq 1$. This can lead to deviations from the alignment limit obtained very differently in the two scenarios considered (e.g., it is seen that even for $\theta=0$, i.e., no mixing between the CP-even Higgs bosons $h$ and $H$, the $h V V$ couplings of C2HDMs deviate from the SM values), yielding totally different phenomenology, both in production and decay of the heavy $H, A$ and $H^{ \pm}$ states. For example, $\Delta \kappa_{V}=-2 \%$ is obtained by $(\xi, \theta)=(0.04,0)$ and $(0,0.2)$ in a C2HDM and E2HDM, respectively. As a result, we can distinguish the two scenarios from the decay Branching Ratios (BRs) of the extra Higgs bosons for a given value of $\Delta \kappa_{V}=\kappa_{V}-1$. Therefore, measurement of a non-zero value of $\Delta \kappa_{V}$ at the LHC would give an indirect evidence for a non-minimal Higgs sector and, by focusing on the same value of $\Delta \kappa_{V}$, we could find that the dominant decay modes of the extra Higgs bosons $H, A$ and $H^{ \pm}$in C2HDMs are different from the E2HDM ones. Similarly, the production cross sections of in C2HDMs and E2HDMs, with the same value of $\Delta \kappa_{V}$, would show significant differences between the elementary and composite cases. Finally, deviations in $\Delta \kappa_{f}$ could guide us in distinguishing amongst the possible types of Yukawa interactions.

As illustrative of this situation, we present Fig. 2, wherein one can see that, in the upper panels (referring to the E2HDM), $H^{ \pm} \rightarrow W^{ \pm} h$ or $H^{ \pm} \rightarrow t \bar{b}$ are dominant for most cases, while, in the lower panels (referring to the $\mathrm{C} 2 \mathrm{HDM}$ ), only $H^{ \pm} \rightarrow t \bar{b}$ is dominant. In fact, in the high mass region of the upper panels, $H^{ \pm} \rightarrow W^{ \pm} h$ becomes the most dominant decay mode, while in the lower panels $H^{ \pm} \rightarrow t \bar{b}$ is the dominant mode in the whole mass region.

\section{Conclusions}

To conclude, we have discovered significant differences on the allowed parameter space of C2HDMs and E2HDMs from perturbative unitarity at the possible energies of the LHC. In addition, possibilities of distinguishing the two sets of scenarios exist herein even if deviations from SM couplings of the SM-like Higgs state are identical in the two cases.

\section{Acknowledgements}

SM and EY are supported in part through the NExT Institute. 

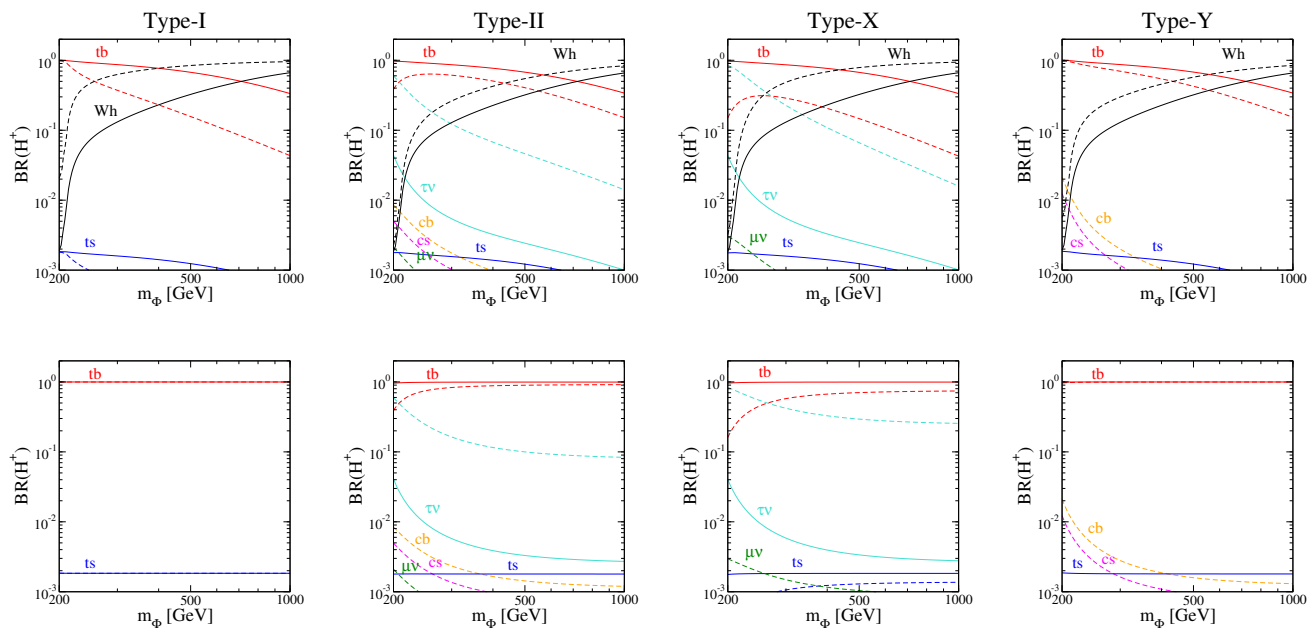

Figure 2: BRs of $H^{ \pm}$as a function of $m_{\Phi}\left(=m_{H}=m_{A}=m_{H^{ \pm}}\right)$with $\tan \beta=3(10)$ for solid (dashed) curves and $M=m_{\Phi}$ in the four types of Yukawa interaction. The upper and lower panels show the case for $\left(s_{\theta}, \xi\right)=(-0.2,0)(\mathrm{E} 2 \mathrm{HDM})$ and $\left(s_{\theta}, \xi\right)=(0,0.04)(\mathrm{C} 2 \mathrm{HDM})$, respectively. Here, $\Delta \kappa_{V}=-2 \%$.

\section{References}

[1] G. Aad et al. [ATLAS Collaboration], Phys. Lett. B 716, 1 (2012).

[2] S. Chatrchyan et al. [CMS Collaboration], Phys. Lett. B 716, 30 (2012).

[3] J. Mrazek, A. Pomarol, R. Rattazzi, M. Redi, J. Serra and A. Wulzer, Nucl. Phys. B 853, 1 (2011).

[4] S. De Curtis, S. Moretti, K. Yagyu and E. Yildirim, Phys. Rev. D 94, 055017 (2016).

[5] S. De Curtis, S. Moretti, K. Yagyu and E. Yildirim, arXiv:1610.02687 [hep-ph].

[6] S. R. Coleman, J. Wess and B. Zumino, Phys. Rev. 177, 2239 (1969);

C. G. Callan, Jr., S. R. Coleman, J. Wess and B. Zumino, Phys. Rev. 177, 2247 (1969).

[7] D. B. Kaplan, Nucl. Phys. B 365, 259 (1991).

[8] M. Aoki, S. Kanemura, K. Tsumura and K. Yagyu, Phys. Rev. D 80, 015017 (2009).

[9] B. W. Lee, C. Quigg and H. B. Thacker, Phys. Rev. D 16, 1519 (1977).

[10] S. Kanemura, T. Kubota and E. Takasugi, Phys. Lett. B 313, 155 (1993).

[11] A. G. Akeroyd, A. Arhrib and E. M. Naimi, Phys. Lett. B 490, 119 (2000).

[12] I. F. Ginzburg and I. P. Ivanov, arXiv:hep-ph/0312374.

[13] I. F. Ginzburg and I. P. Ivanov, Phys. Rev. D 72, 115010 (2005).

[14] S. Kanemura and K. Yagyu, Phys. Lett. B 751, 289 (2015).

[15] J. F. Gunion, H. E. Haber, G. L. Kane and S. Dawson, Front. Phys. 80, 1 (2000). 